\title{
The SIDER2 elements, interspersed repeated sequences that populate the Leishmania genomes, constitute subfamilies showing chromosomal proximity relationship Jose M Requena*1, Cristina Folgueira ${ }^{1}$, Manuel C López ${ }^{2}$ and M Carmen Thomas ${ }^{2}$
}

\author{
Address: ${ }^{1}$ Centro de Biología Molecular "Severo Ochoa" (CSIC-UAM), Universidad Autónoma de Madrid, 28049 Madrid, Spain and \\ 2Departamento de Biología Molecular, Instituto de Parasitología y Biomedicina "López-Neyra", CSIC, 18000 Granada, Spain \\ Email: Jose M Requena* - jmrequena@cbm.uam.es; Cristina Folgueira - cfolgueira@cbm.uam.es; Manuel C López - mclopez@ipb.csic.es; M \\ Carmen Thomas - mcthomas@ipb.csic.es \\ * Corresponding author
}

Published: 2 June 2008

BMC Genomics 2008, 9:263 doi:10.1186/147I-2164-9-263

This article is available from: http://www.biomedcentral.com/I47I-2/64/9/263

(C) 2008 Requena et al; licensee BioMed Central Ltd.

This is an Open Access article distributed under the terms of the Creative Commons Attribution License (http://creativecommons.org/licenses/by/2.0), which permits unrestricted use, distribution, and reproduction in any medium, provided the original work is properly cited.

\begin{abstract}
Background: Protozoan parasites of the genus Leishmania are causative agents of a diverse spectrum of human diseases collectively known as leishmaniasis. These eukaryotic pathogens that diverged early from the main eukaryotic lineage possess a number of unusual genomic, molecular and biochemical features. The completion of the genome projects for three Leishmania species has generated invaluable information enabling a direct analysis of genome structure and organization.
\end{abstract}

Results: By using DNA macroarrays, made with Leishmania infantum genomic clones and hybridized with total DNA from the parasite, we identified a clone containing a repeated sequence. An analysis of the recently completed genome sequence of $L$. infantum, using this repeated sequence as bait, led to the identification of a new class of repeated elements that are interspersed along the different $L$. infantum chromosomes. These elements turned out to be homologues of SIDER2 sequences, which were recently identified in the Leishmania major genome; thus, we adopted this nomenclature for the Leishmania elements described herein. Since SIDER2 elements are very heterogeneous in sequence, their precise identification is rather laborious. We have characterized 54 LiSIDER2 elements in chromosome 32 and 27 ones in chromosome 20. The mean size for these elements is $550 \mathrm{bp}$ and their sequence is $\mathrm{G}+\mathrm{C}$ rich (mean value of $66.5 \%$ ). On the basis of sequence similarity, these elements can be grouped in subfamilies that show a remarkable relationship of proximity, i.e. SIDER2s of a given subfamily locate close in a chromosomal region without intercalating elements. For comparative purposes, we have identified the SIDER2 elements existing in L. major and Leishmania braziliensis chromosomes 32. While SIDER2 elements are highly conserved both in number and location between $L$. infantum and $L$. major, no such conservation exists when comparing with SIDER2s in L. braziliensis chromosome 32.

Conclusion: SIDER2 elements constitute a relevant piece in the Leishmania genome organization. Sequence characteristics, genomic distribution and evolutionarily conservation of SIDER2s are suggestive of relevant functions for these elements in Leishmania. Apart from a proved involvement in posttrancriptional mechanisms of gene regulation, SIDER2 elements could be involved in DNA amplification processes and, perhaps, in chromosome segregation as centromeric sequences. 


\section{Background}

Repetitive DNA sequences constitute a substantial proportion of eukaryotic genomes. For example, in mammals they account for nearly half of the genome, and in some plants they constitute up to $90 \%$ of the genome [1]. Most of these repeated DNAs are, or were originated from, transposable elements (TEs, also known mobile elements) through transposing and duplicating events. On the basis of mechanisms of their transposition, TEs can be divided into two classes: retrotransposons, which proliferate via reverse transcription, and DNA transposons, which move strictly through DNA intermediates. Frequently, genomes harbour few active TEs; instead, genomes contains multiple repetitive elements representing remnants (or dead elements) derived from TEs [2]. Although repetitive DNA elements have been often considered as "selfish" or "parasitic" DNAs, the now growing evidence is that these elements are involved in shaping genomes and are playing important role in epigenetic regulation of genome expression $[1,3]$.

Protozoan parasites of the genus Leishmania are causative agents of a complex of diseases known as leishmaniasis. The burden associated with these diseases remains important: 1.5-2 million new cases per year and 350 million people at risk in 88 countries [4]. Apart from its impact in human health, Leishmania parasites and related trypanosomes (i.e. Trypanosoma cruzi and Trypanosoma brucei) are being extensively studied because of peculiar molecular and cellular characteristics. The genome of Leishmania major was sequenced [5], and more recently the genome sequences for two other Leishmania species (Leishmania infantum and Leishmania braziliensis) have been also deciphered [6]. The comparison of these sequences reveals marked conservation of the genome architecture within the Leishmania genus, showing similar gene content and a remarkable degree of synteny [7]. The organization of protein-coding genes into long, strand-specific, polycistronic clusters is a conspicuous feature of the Leishmania species, also observed in the T. brucei and T. cruzi genomes [8]. This peculiar gene organization seems to be related to the lack of transcriptional control by RNA polymerase II promoters; rather, transcription initiation appears to begin in a low fidelity manner transcribing long polycistronic precursor transcripts [9]. Despite having diverged 200 to 500 million years ago, the genomes of L. major, T. brucei and T. cruzi are highly synthenic. For example, 68 and $75 \%$ of the genes in T. brucei and L. major remain in the same gene order [8]. In spite of this conservation in chromosome organization, the genomes of these trypanosomes differ in the content of repeated sequences. Unlike Leishmania, the genomes of T. brucei and T. cruzi are riddled with interspersed elements [10-12].
The Leishmania genome is relatively poor in repeated sequences. The first repetitive DNA sequence characterized in Leishmania corresponded to the telomeric repeats [13]. Afterwards, multiple tandem repeats of a 60-bp sequence, named Lmet2, were found on at least six chromosomes of parasites of the L. donovani complex, being absent from other Leishmania species [14]. Piarroux et al [15] characterized a low copy, repetitive DNA sequence from $L$. infantum that was located exclusively at a large chromosome; this sequence was detected in many other Leishmania species. A repeated sequence with features of minisatellite DNA was characterized in the L. infantum genome; this element, called LiSTIR1, is 81-bp long and $\mathrm{G}+\mathrm{C}$ rich and it was found interspersed at the subtelomeric regions of four chromosomes [16]. A 348-bp long element, designated LiR3, was found tandemly repeated within the non-transcribed spacers of the rDNA locus of $L$. infantum [17]. Conserved repeats, named LCTAS, have been characterized to be adjacent to telomeres in $L$. braziliensis, L. major, L. mexicana and L. lainsoni [18]. Also, several subtelomeric repetitive sequences have been characterized, showing to be responsible for size differences among the three L. major homologues for chromosome 1 [19]. Similar repeats have been found as tandemly arranged clusters at subtelomeric regions in chromosomes 1,19 and 22 of $L$. infantum. Interestingly, these repeats are transcribed by RNA polymerase II into noncoding RNAs in a developmentally regulated manner [20]. Non-LTR retrotransposons are abundant in the genome of $T$. brucei and T. cruzi; by contrast, retroelements are absent from the L. major genome, where only remnants of degenerated ingi/L1Tc-related elements (or DIREs) are detectable (the L. major haploid genome contains 52 DIREs). Evolutionary analyses indicate that the trypanosomatid ancestor contained active transposable elements that have been retained in the genus Trypanosoma, but were lost in the $L$. major evolutionary line [21]. Recently, in an outstanding work, Bringaud et al [22] have found that the L. major contains two classes of short interspersed repeated sequences, SIDER1 (785 copies) and SIDER2 (1073 copies), which displays hallmarks of trypanosomatid retroposons. Members of the SIDER1 family show high sequence similarity with a conserved 450-550-bp element, located in the 3'UTR of several Leishmania amastigote-specific transcripts, that is implicated in stage-specific translational control $[23,24]$. SIDER2 elements, also located predominantly within 3'UTRs, have a demonstrated role in mRNA degradation [22]. Thus, it was postulated that Leishmania have recycled the retroposon remnants to regulatory sequences to globally modulate the expression of a number of genes [22].

In the course of studying repetitive DNA in the L. infantum genome, we identified and characterized a family of repeated sequences, which are interspersed along the dif- 
ferent chromosomes. These sequence elements are present in different Leishmania species and, here, we show a detailed analysis of these elements in the L. infantum chromosomes 20 and 32, and in the L. braziliensis and L. major chromosome 32. During the preparation of this manuscript, the existence of this class of sequences in the $L$. major genome was reported [22], and, consequently, we adopted the proposed name (SIDER, Short Interspersed Degenerated Retroposon) for the elements identified in this work.

\section{Results \\ Identification of a new family of repeated sequences in $L$. infantum}

As an approach to isolate and identify repetitive sequences in the L. infantum genome, we hybridized genomic DNA macroarrays of L. infantum (JPC strain) with labelled total genomic DNA of this parasite. A clone, named pGLi5G8g, was selected for further analysis on the basis of its strong hybridization signal. Sequence analysis showed that the 2280-bp long insert locate on L. infantum chromosome 32 (EMBL accession number AM937229). However, the most striking observation, derived from the BLAST analysis, was that sequences, homologous to the 5 '-end region of this clone, were also present in many additional locations in all the $36 \mathrm{~L}$. infantum chromosomal contigs. A thoughtful search along the L. infantum chromosome 32 (contig LinJ32_20070420_V3; [25]), using iterative rounds of BLAST searches, led us to the identification up to 54 sequence elements. We named these elements as LiSIDER2s, following the nomenclature coined by Bringraud and coworkers in a recent publication describing the existence of this class of sequences in the L. major genome; SIDER stands for short interspersed degenerated retroposon [22]. The different LiSIDER2s found in chromosome 32 are listed in Table 1 . These elements have two salient features: a size around $550 \mathrm{bp}$ and a high $\mathrm{G}+\mathrm{C}$ content (mean value 66.5\%). Based on the $L$. infantum database (GeneDB), we have calculated that the $\mathrm{G}+\mathrm{C}$ content for the L. infantum chromosome 32 is 58.8 , very similar to the $\mathrm{G}+\mathrm{C}$ content for the whole $L$. infantum genome [7]. All LiSIDER2 elements have $\mathrm{G}+\mathrm{C}$ content higher than the mean value for the entire genome, and some of them exceed $70 \%$. A physical location of the LiSIDER2 elements on L. infantum chromosome 32 is shown in figure 1. These elements were found in both plus and minus strands of the chromosome and they showed a quite even distribution along the chromosome. However, it is noticeable that most of the elements have the same orientation as the polycistronic transcription units in which they are located.

Phylogenetic analyses (Fig. 2), based on the ClustalW alignment of the different LiSIDER2s [see Additional file 1], allowed us to group these elements into subfamilies. A subfamily was defined as a group of LiSIDER2s sharing sequence identity $\geq 85 \%$. Thus, the 54 LiSIDER2s can be grouped into 13 subfamilies (named A to $M$ ), remaining 9 orphan elements (Table 1; Fig. 2). Remarkably, members of a given subfamily show a relationship of proximity, i.e. they are grouped close in the chromosome without intercalating non-familiar LiSIDER2s (Fig. 1). For example, elements of the subfamily A, composed of eight members, are located at the left hand of L. infantum chromosome 32 and no at other chromosomal regions.

Another structural feature of LiSIDER2 elements, evidenced during the bioinformatics identification of the elements, was their composite nature. Thus, the elements from different subfamilies share only sequence blocks of variable size that are present in different combinations in each LiSIDER2. An example illustrating this observation is shown in Figure 3A. Nevertheless, a conserved consensus sequence for the LiSIDER2s can be derived from the alignment of the 54 elements present in chromosome 32 (Fig. $3 \mathrm{~B})$, suggesting a common origin for all elements. As suggested by Bringaud et al. [22], SIDER2 elements could be vestigial retroposons, derived from non-LTR retrotransposons of the ingi/L1Tc clade that remains active in the genomes of T. brucei and T. cruzi [21]. This hypothesis is based mainly upon the existence at the 5'-extremity of some LmjSIDER2 elements of the "79-bp signature", which constitutes the hallmark of trypanosomatid nonLTR retrotransposons and related elements [26]. Using the two "79-bp signatures" found in the L. major SIDER2 elements (LmSIDER2a and LmSIDER2b, [22]) for BLASTN searches, we found 35 matches in the L. infantum chromosome 32 sequence. Interestingly, 34 out of the 35 matches were coincident with the location of LiSIDER2 elements, indicating that this is not a fortuitous association. Thus, $34(63 \%)$ out of the 54 SIDER2 elements, present in $L$. infantum chromosome 32, have a distinguishable "79-bp signature" that invariantly is located at, or close to, the 5 'end of the element. For most of the LiSIDER2, the "79bp signature" was found to be more similar to the LmSIDER2b sequence than to the LmSIDER2a one (Table 1). A comparison of the consensus "79-bp signature" present in the LiSIDER2s with that existing in other trypanosomatid elements is shown in figure 3C. For some LmjSIDER2 elements (18.9\%), the presence of putative target site duplication (TSD) was noticed by Bringaud and co-workers [22]. However, after inspection of sequences immediately upstream and downstream of the different LiSIDER2s in chromosome 32, we did not find clear TSD sequences, even though when members of a subfamily were separately analyzed. Also, the presence of short adenosine-rich stretches was described at the 3'-end of some of the LmjSIDER2 elements. In the characterized LiSIDER2, adenosine runs were found to be present in 
Table I: SIDER2 elements present in the $L$. infantum chromosome 32 and comparison with their homologues in $L$. major

\begin{tabular}{|c|c|c|c|c|c|c|}
\hline \multicolumn{4}{|c|}{ L. infantum SIDER2 element } & \multicolumn{3}{|c|}{ L. major SIDER2 homologue } \\
\hline Name $^{a}$ & Subfamily & Size $(\% \mathbf{G}+\mathbf{C})$ & 79-bp signature ${ }^{b}$ & Namec & Size $(\% \mathbf{G}+\mathbf{C})$ & Sequence identity (\%) \\
\hline $32-18439 r$ & $A$ & $560(68.4)$ & $2 b$ & $32-16072 r$ & $546(67.4)$ & 83 \\
\hline $32-30662 r$ & $A$ & $560(68.9)$ & $2 b$ & $32-28347 r$ & $549(67.2)$ & 82 \\
\hline $32-39696 d$ & A & $570(70.4)$ & $2 b$ & $32-37464 d$ & $54 I(64.3)$ & 69 \\
\hline $32-46425 r$ & $A$ & $570(70.0)$ & $2 a$ & $32-44074 r$ & $547(67.8)$ & 81 \\
\hline $32-72349 d$ & $A$ & $568(70.4)$ & $2 b$ & $32-70108 d$ & $550(69.3)$ & 82 \\
\hline $32-84516 r$ & $A$ & $546(70.5)$ & $2 b$ & $32-82363 r$ & $550(68.7)$ & 81 \\
\hline $32-121058 r$ & $A$ & $550(69.6)$ & $2 b$ & $32-119100 r$ & $518(64.5)$ & 76 \\
\hline $32-127617 r$ & $A$ & $515(69.7)$ & $2 b$ & $32-125634 r$ & $515(63.5)$ & 74 \\
\hline $32-169517 r$ & B & $564(64.0)$ & $2 a$ & $32-16808 \mathrm{Ir}$ & $568(64.1)$ & 87 \\
\hline $32-185886 r$ & B & $564(64.2)$ & $2 a$ & $32-184597 r$ & $569(64.2)$ & 87 \\
\hline $32-222503 d$ & C & $594(66.3)$ & $2 b$ & $32-221358 d$ & $555(68.7)$ & 84 \\
\hline $32-23297$ Id & C & $578(67.0)$ & $2 b$ & $32-231497 d$ & $582(69.1)$ & 85 \\
\hline $32-253342 d$ & C & $578(67.5)$ & $2 b$ & $32-252118 d$ & $578(68.9)$ & 86 \\
\hline $32-311078 r$ & & $535(61.7)$ & $2 b$ & $32-308193 r$ & 601 (59.6) & 79 \\
\hline $32-354985 d$ & $D$ & $586(63.5)$ & $2 b$ & $32-351852 d$ & $574(62.5)$ & 87 \\
\hline $32-367858 d$ & $\mathrm{D}$ & $590(63.6)$ & $2 b$ & $32-364746 d$ & $574(62.9)$ & 86 \\
\hline $32-42091 \mathrm{Id}$ & $E$ & $540(68.0)$ & & $32-4 \mid 363$ Id & 561 (67.7) & 84 \\
\hline $32-456060 d$ & $E$ & $556(67.8)$ & & $32-448879 d$ & $566(67.3)$ & 85 \\
\hline $32-460150 r$ & $E$ & $555(67.8)$ & & $32-452988 r$ & $562(67.6)$ & 86 \\
\hline $32-520410 d$ & $\mathrm{~F}$ & $661(68.4)$ & & $32-515291 d$ & $663(70.7)$ & 78 \\
\hline $32-542863 d^{*} *$ & $\mathrm{~F}$ & $422(68.5)$ & & $32-537679 d$ & 579 (7I.7) & 59 \\
\hline $32-546470 r$ & $\mathrm{~F}$ & $679(68.3)$ & & $32-54 \mid 585 r$ & 662 (71.0) & 77 \\
\hline $32-588727 r$ & $\mathrm{~F}$ & $588(66.7)$ & & $32-584100 r$ & $528(70.6)$ & 73 \\
\hline $32-598168 r$ & $\mathrm{~F}$ & 597 (68.2) & & $32-593579 r$ & $565(69.4)$ & 80 \\
\hline $32-642986 d^{*} *$ & $\mathrm{~F}$ & $46 I(68.6)$ & & $32-638510 d$ & $542(68.3)$ & 62 \\
\hline $32-661708 r$ & & $552(64.7)$ & $2 b$ & $32-657337 r$ & $404(62.8)$ & 62 \\
\hline $32-726312 r$ & G & 490 (66.9) & $2 b$ & $32-72284 I r$ & $453(66.7)$ & 83 \\
\hline $32-734536 r$ & G & $489(68.5)$ & $2 b$ & $32-731206 r$ & $556(66.7)$ & 73 \\
\hline $32-752616 r$ & G & $492(67.9)$ & $2 b$ & $32-749267 r$ & $540(66.7)$ & 78 \\
\hline $32-755765 r$ & G & 491 (68.8) & $2 b$ & $32-752467 r$ & $553(66.2)$ & 75 \\
\hline $32-769406 r$ & & $44 I(61.0)$ & & $32-766 \mid 45 r$ & $569(57.8)$ & 69 \\
\hline $32-772687 r$ & & 425 (60.9) & & $32-769534 r$ & $408(60.5)$ & 80 \\
\hline $32-775723 r^{*}$ & $\mathrm{H}$ & $265(65.3)$ & & $32-772586 r^{d}$ & $430(66.5)$ & 54 \\
\hline $32-79440 \mathrm{Ir}$ & $\mathrm{H}$ & $603(63.2)$ & $2 b$ & $32-790497 r$ & 577 (62.9) & 86 \\
\hline $32-802492 r$ & $\mathrm{H}$ & $550(63.8)$ & $2 b$ & $32-798632 r$ & $54 I(64.5)$ & 86 \\
\hline $32-808837 r$ & $\mathrm{H}$ & $550(64.0)$ & $2 b$ & $32-804952 r$ & $54 I(64.7)$ & 87 \\
\hline $32-827594 d$ & $\mathrm{H}$ & $591(62.4)$ & $2 b$ & $32-824177 d$ & $581(63.0)$ & 91 \\
\hline $32-881720 r$ & 1 & $570(66.7)$ & $2 b$ & $32-8779 \mid 3 r$ & $581(65.4)$ & 90 \\
\hline $32-890515 r$ & 1 & $573(66.8)$ & $2 b$ & $32-886987 r$ & $584(65.2)$ & 90 \\
\hline $32-919839 r$ & $J$ & $623(70.0)$ & & $32-916263 r$ & $502(69.5)$ & 74 \\
\hline $32-937896 d$ & $\mathrm{~J}$ & $507(70.6)$ & & $32-934324 d$ & $600(69.7)$ & 75 \\
\hline $32-953057 r$ & $\mathrm{~J}$ & $623(70.0)$ & & $32-949068 r$ & $600(69.5)$ & 88 \\
\hline $32-991979 r$ & $\mathrm{~K}$ & $614(65.3)$ & $2 b$ & $32-988462 r$ & $594(65.3)$ & 86 \\
\hline $32-1017837 r$ & $\mathrm{~K}$ & $614(65.2)$ & $2 b$ & $32-1014737 r$ & $594(65.3)$ & 85 \\
\hline $32-1|2807| r$ & & $506(63.4)$ & $2 b$ & $32-1125938 r$ & $519(62.0)$ & 88 \\
\hline $32-1|4| 454 r$ & & $404(60.4)$ & & $32-1167639 d$ & $367(61.6)$ & 52 \\
\hline $32-1172151 d$ & $\mathrm{~L}$ & $506(67.8)$ & & $32-1169025 d^{d}$ & $367(70.6)$ & 67 \\
\hline $32-1195026 d$ & $\mathrm{~L}$ & $602(66.5)$ & & $32-1191646 d$ & $618(68.0)$ & 87 \\
\hline $32-1219109 r$ & $\mathrm{~L}$ & $602(66.6)$ & & $32-1215657 r$ & $618(67.8)$ & 89 \\
\hline $32-1284330 d$ & & 458 (65.9) & $2 b$ & $32-1278797 d$ & 559 (63.9) & 74 \\
\hline $32-1405232 d$ & & 467 (64.9) & $2 b$ & $32-1399746 d$ & 468 (63.9) & 74 \\
\hline $32-1496 \mid 87 d$ & $M$ & $497(66.4)$ & $2 a$ & $32-1509833 d$ & $4 I I(66.4)$ & 60 \\
\hline $32-1532945 d^{*}$ & $M$ & $365(68.2)$ & & $32-1546424 d$ & $409(65.8)$ & 79 \\
\hline $32-1545347 d$ & & $513(61.6)$ & $2 b$ & $32-1558844 d$ & $457(62.1)$ & 68 \\
\hline
\end{tabular}

aThe elements are named with the chromosome number (i.e., 32) and the position in the chromosome, according to the contig LinJ32_20070420_V3 sequence; $d$ and $r$ denote that the element is located in the plus or minus DNA strand, respectively. The indicated nucleotide corresponds to either the start or end of the element, depending on its location in the plus or minus DNA strand, respectively. bThe presence of significant BLASTN matches with the "79-bp signature" LmSIDER2a (2a) or LmSIDER2b (2b) is indicated. 'The elements are named with the chromosome number (i.e., 32) and the position according to the contig LmjF32_0I_2005060I_V5.2 sequence; $d$ and $r$ denote that the element is located on the plus or minus strand, respectively.

*Truncated element. 
about $28 \%$ of the elements, either at the 3 '-end or in close proximity to it.

In order to know whether or not this peculiar organization of LiSIDER2 elements is shared by the elements located in other $L$. infantum chromosomes, we carried out a systematic search of LiSIDER2s along the chromosome 20 . We chose this chromosome, because we realized that sequences similar to LiSIDER2s had been previously described in the homologue chromosome in L. major [27]. As shown in Table 2, 27 elements were identified in the L. infantum chromosome 20. Similarly, these LiSIDER2s were found to have $\mathrm{G}+\mathrm{C}$-rich sequences, to have a size around 500-bp, and can be grouped in subfamilies according to sequence homology ( $N$ to $S$ ). In chromosome 20, we found that members of subfamilies $\mathrm{Q}$ and $\mathrm{R}$ are intercalated (Fig. 4A); however, it should be noted that these subfamilies are closely related each other in sequence (Fig. 4B; [see Additional file 2]). Another relevant finding was that two LiSIDER2s, which constitute subfamily S (Table 2), have an uncommon size (1270bp), being the SIDER2-homologue region located at the 3'-end half of these elements. As occurred with LiSIDER2s of chromosome 32, most of LiSIDER2s in chromosome 20 are in the same orientation as the transcriptional units (Fig. 4A). Furthermore, 15 out of the 27 (56\%) LiSIDER220 have a distinctive "79-bp signature" (Table 2).

As deduced from BLAST analyses (data not shown), the rest of $\mathrm{L}$. infantum chromosomes must be also populated by LiSIDER2 elements showing similar features as those described in chromosomes 20 and 32. Taking into account both the chromosomal size and the number of SIDER2s found in L. infantum chromosomes 20 and 32, we estimated that the L. infantum haploid content of SIDER2s would be around 1150 copies. This estimation is in agreement with the determination of 1073 copies of LmjSIDER2 in the L. major genome [22].

\section{Sequences homologous to LiSIDER2s are also present in the genome of other Leishmania species}

Since the complete sequence of the L. major is known [5], we carried out the same bioinformatics analysis on the $L$. major database using as query sequences the different LiSIDER2 elements found in the L. infantum chromosome 32 (Table 1). In all cases, the best scores were observed with sequences located in the L. major chromosome 32 . Table 1 summarizes molecular features of the SIDER2s found in the L. major chromosome 32. Remarkably, it was observed an extremely high conservation, both in sequence and genomic location, of the SIDER2s found in the L. major and L. infantum chromosomes 32 . To avoid confusion, following the genetic nomenclature directions for kinetoplastids [28], we named the L. major elements as LmjSIDER2. In an independent study, Bringaud et al [22] identified 55 SIDER2s elements in the L. major chromosome. Except for small variations in the coordinates, there was a total correspondence between the 54 elements identified by us (Table 1) and those identified by Bringaud and colleagues. Our analysis failed to find the LmjSIDER2 starting at position 626445 [22].

Recently, the completion of the L. braziliensis genome sequence has been announced [6], and we considered of interest to search for the existence of these elements in this species. First analyses indicated that SIDER2 sequences indeed exist in the L. braziliensis genome, but the distribution of the elements in the chromosome 32 was not conserved regarding the conspicuous conservation of SIDER2 elements that exists between $L$. infantum and $L$. major chromosome 32. Thus, BLAST searches using the LiSIDER2 sequences from chromosome 32 showed that best scores were not with sequences from $L$. braziliensis chromosome 32. Rather, bestfits for each LiSIDER2-32 sequence were found with sequences distributed among the different $L$. braziliensis chromosomes, indicating that SIDER2s are not chromosome specific for all Leishmania species. However, the intrachromosomal organization of these elements in the L. braziliensis genome showed features similar to that found in the other two Leishmania species. Thus, most of the 48 LbSIDER2 elements, which

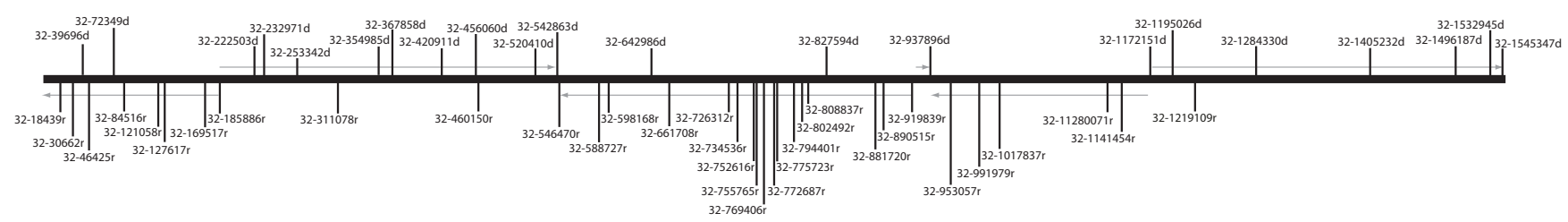

\section{Figure I}

Genomic organization of SIDER2 elements in the $L$. infantum chromosome 32 . The position and the orientation of the elements were deduced from the sequence of contig Linj32_20070420_V3 [25]. The extent and directions of the transcriptional units are denoted by horizontal grey arrows. See table I for further details of the SIDER2 elements mapping to this chromosome. 


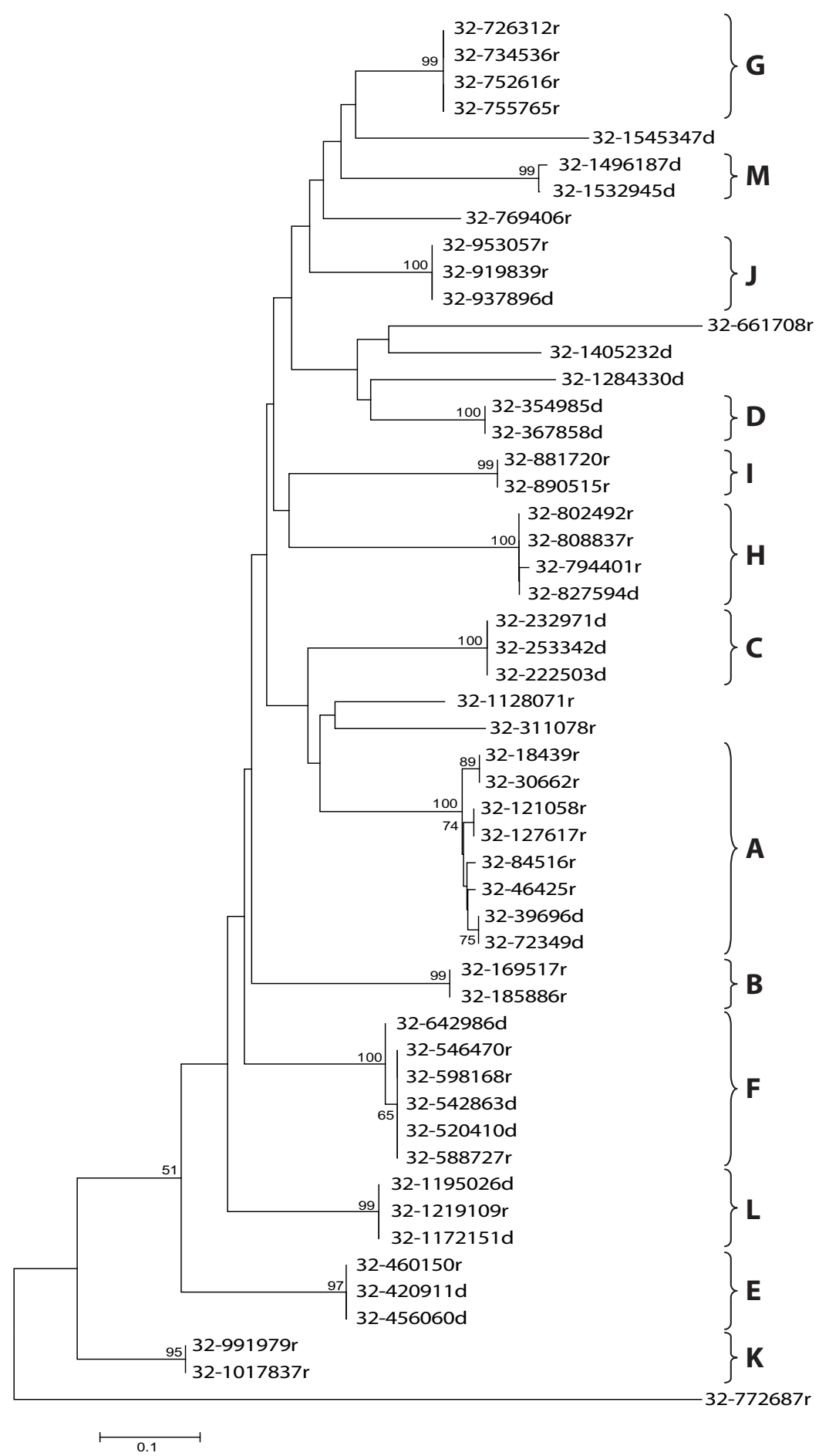

Figure 2

Clustering by phylogenetic analysis of SIDER2 elements found in the L. infantum chromosome 32 . The alignment of the SIDER2 sequences was produced by ClustalW [see Additional file I]. The phylogenetic tree was constructed with the program MEGA version 3.1 using the Neighbour-Joining method. The elements 32-775723r and 32-1 I4I454r were excluded from the phylogenetic analysis because the program failed when calculating the Kimura distances for these sequences. Bootstrap values, expressed as percentages, were obtained from 1000 replicates. Only values higher than 50 are shown. The positions of the different subfamilies (A-M) are shown in the right margin. The scale represents number of changes per nucleotide. 
A

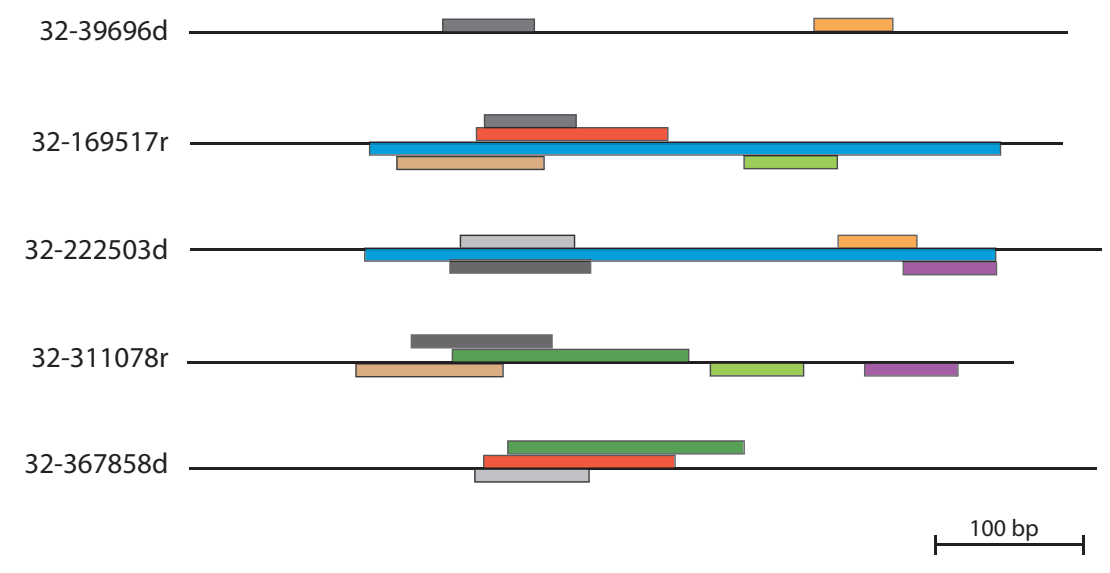

B

\begin{tabular}{ll} 
NCCNCNNNGCGTGGTNTCTCAGGGNCCAGTNCTCCNNNNNCNNTCTNNGTG & 51 \\
GNGAAGCCNNGCNGCCCCTCCTNNCCNNCCNAATGCCGAGCACCTCTGG & 102 \\
TGGTGACAGGGTCAGGCGCCTACGACGTNGGGGGAGGTCAGAGCGATGCAT & 153 \\
\hline CGCTGCTGATGTCGGCGGTCAGGTCCTGGATGGCGTTGCGTCGGGCGACC & 204 \\
TGCGACAGTGAACACGCTTGTGCCATCCATATGATTTGGCAGAGTGTCAG & 255 \\
CGTGGCTCGAGCGTATCCCNCCCCCCGGCCCTCNCACTGCCCACTGGTGT & 306 \\
GGGGAGCCNGAGCCANNCCGNGGGGGGGGCGCACCGNGTGGCGNCCGGCAT & 357 \\
GGAGGGGGNAGCGGCNGNGAGGCGGCCTGCGAGGCGGGGGGTGGGNNGGGT & 408 \\
AGAGTTTGAGGCAGNGGCCGTGCTCNGATGACTGAGTCGGCGCATTGCTGC & 459 \\
AACGCGTGTCTACCGCTGCTTCGCACCACGCGATGTGGGCCTGTGACAGGC & 510 \\
CGGGNNNNNNNGGNNGNGNGGNGNNNNNNNNNTGNNNNNGNGNNNN & 556
\end{tabular}

RIME CCCTGGCGATGCCGGCCACCTCAACGTGGTGCCAGGGTCCAGTACCCCGTATCATCGGGGGAAGCCAAGAGCCAGCAGC 79 NARTC CCCTGGCTCAGCCGGCCACCTCAACGTGGTGCCAGGGTCTAGTACTCTTTGCTA-GAGAGGAAGCTAAGCGCCTGCTGC 78 LmSIDER2a CCCTGATGACGAGGGACACCTCAGCGTGGTATCAGGGTCCAGTACACCCCACTCTGTGAGGAAGCCGAGCAGCTCCCTC 79 LmSIDER2b CCCTGCCAATGCCGAACCACTTCTGGTGGTGACAGGGTCCAGTGCCTACTACGT-AGGGGAAGGTCAGAGCGATGCATC 77 LiSIDER2 CCNNCCNAATGCCGAGCCACCTCTGGTGGTGACAGGGTCAGGCGCCTACGACGT-NGGGGGAGGTCAGAGCGATGCATC 78

\section{Figure 3}

Structural features of LiSIDER2 elements. (A) The sequence homology between LiSIDER2s elements from different subfamilies is restricted to segments of varying sizes. Pairwise alignments among the indicated LiSIDER2s sequences were obtained using the BLAST two sequences tool available at the NCBI Web Page [45]. The retrieved matches are depicted by coloured boxes. (B) The 556-nucleotide long consensus sequence was derived from the ClustalW alignment of the 54 LiSIDER2 sequences identified in chromosome 32 . A nucleotide was given a consensus status when was present in at least $50 \%$ of the sequences. Positions conserved in more than $60 \%$ of the sequences are shaded in red, and those conserved in more than $80 \%$ of the sequences are shaded in green. The underlined sequence corresponds to the "79-bp signature" sequence of trypanosomatid retroposons. (C) Comparison of the "79-bp signatures" present in the T. brucei RIME element, T. cruzi NARTc element, LmjSIDER2 elements (LmSIDER2a and LmSIDER2b), and LiSIDER2-32 consensus sequence. Conserved residues are boxed and shaded in grey; gaps (-) were introduced to maximize the alignments.

were identified in the L. braziliensis chromosome 32, can be grouped, according to sequence homology, in subfamilies (a to k), whose members also show a relationship of proximity (Table 3 ).
In addition to the analysis of Leishmania genome databases, we performed searches looking for SIDER2 homologue elements in general databases (EMBL and GenBank). A large number of entries were retrieved; how- 
Table 2: SIDER elements present in the $L$. infantum chromosome 20

\begin{tabular}{|c|c|c|c|}
\hline Name $^{a}$ & Subfamily & Size (\%GC) & 79-bp signature \\
\hline $20-315 r$ & $\mathrm{~N}$ & $477(66.3)$ & $2 b$ \\
\hline $20-98 \mid 5 r$ & $\mathrm{~N}$ & $554(66.3)$ & $2 b$ \\
\hline $20-26129 r$ & $\mathrm{~N}$ & $526(65.8)$ & $2 b$ \\
\hline $20-38171 r^{*}$ & $N$ & $356(67.4)$ & \\
\hline $20-104446 d$ & 0 & $576(65.8)$ & $2 a$ \\
\hline $20-112062 r$ & 0 & $571(65.5)$ & $2 a$ \\
\hline $20-121152 d^{*}$ & 0 & $425(65.2)$ & \\
\hline $20-133376 d$ & 0 & $661(64.2)$ & $2 a$ \\
\hline $20-172973 d$ & 0 & $651(64.7)$ & $2 a$ \\
\hline $20-208609 r$ & & $384(63.8)$ & $2 b$ \\
\hline $20-248078 r$ & & $378(61.4)$ & $2 b$ \\
\hline $20-33452$ Id & & $221(58.8)$ & \\
\hline $20-4 \mid 8908 d$ & & $466(65.7)$ & $2 a$ \\
\hline $20-432767 r$ & $P$ & $561(65.4)$ & \\
\hline $20-441039 d$ & $P$ & $592(65.0)$ & \\
\hline $20-449285 d$ & $\mathrm{P}$ & $603(65.2)$ & \\
\hline $20-48306 \mathrm{Ir}$ & $Q$ & $466(67.4)$ & \\
\hline $20-510191 r^{*}$ & Q & $253(70.0)$ & \\
\hline $20-5|23| 7 d$ & $\mathrm{R}$ & $451(66.3)$ & $2 b$ \\
\hline $20-517565 r$ & Q & 334 (71.9) & \\
\hline $20-520085 d$ & $\mathrm{R}$ & $444(66.4)$ & $2 b$ \\
\hline $20-543749 d$ & $\mathrm{R}$ & $455(65.5)$ & $2 b$ \\
\hline $20-550589 d$ & $\mathrm{R}$ & $455(65.5)$ & $2 b$ \\
\hline $20-575257 d$ & $Q$ & $468(68.6)$ & $2 b$ \\
\hline $20-609548 d$ & $\mathrm{~S}$ & $1270(70.0)$ & \\
\hline $20-616384 d$ & $S$ & $1270(69.8)$ & \\
\hline $20-716639 r$ & & $556(58.6)$ & \\
\hline
\end{tabular}

aThe elements are named with the chromosome number (i.e., 32) and the position in the chromosome, according to the contig Linj20_20070420_V3 sequence; $d$ and $r$ denote that the element is located in the plus or minus DNA strand, respectively.

bThe presence of significant BLASTN matches with the "79-bp signature" LmSIDER2a (2a) or LmSIDER2b (2b) is indicated.

*Truncated element.

ever, all entries contained Leishmania sequences and homologous sequences were not found in other organisms, with an intriguing exception. Thus, we found a significant homology between LiSIDER-32-121058d and the EMBL entry with accession number AM094505, which corresponds to a Lutzomyia longipalpis EST clone NSFM162h01. Remarkably, this sandfly species acts as Leishmania transmission vector. On the other hand, BLAST searches in the T. cruzi and T. brucei genome databases (GeneDB) yielded not results, indicating that these elements are specific for the Leishmania genus. Among the retrieved entries from the EMBL and GenBank databases, there are sequences derived from L. amazonensis ( $\underline{\mathrm{U} 70540}$, AB029444, AY427440S3, DQ092336), L. braziliensis (DQ092335), L. donovani (Z94053, AC093553, $\underline{\mathrm{AF} 067495}, \quad$ AF109296, $\quad$ AY028171, AY791850, DQ092337), L. hoogstraali (DQ092338), L. infantum (93416, L27052, AJ628942, AM118098), L. major (Z54138, AY227807, AY328521, AY491007), L. mexicana (Z46971， AF350492， AJ131960， AJ427448， AJ548776,
AY170465), and L. tarentolae (AY842846). Remarkably, there exist many entries corresponding to $L$. chagasi cDNAs (V669830, CV667316, CV670663, CV663048, CV669851， CV669636， CV662260， CV666468， CV669797， CV666868， CV664167， CV669564， CV665051, CV663324, CV668078, CV668316) that have significant BLAST scores with SIDER2 sequences.

The bioinformatics analysis indicated that SIDER2 elements are widespread among the different Leishmania species. In order to obtain experimental evidence, Southern blots containing Sall-digested genomic DNA from $L$. infantum, L. major, L. tropica, L. mexicana and L. braziliensis were probed with two different LiSIDER2s, LiSIDER2-32121058r and LiSIDER2-20-575257d (Fig. 5). Complex hybridization patterns were obtained with each one of the probes, confirming the repeated nature of the SIDER2 elements. The hybridizations patterns are also in agreement with a scattered distribution of these elements in the Leishmania genome. Although, differences were observed in the signal intensity of particular bands among the different Leishmania species, the global hybridization signal was found very similar, suggesting that a similar number of SIDER2s elements must be present in the different species tested.

\section{Discussion}

In a recent work, Bringaud and co-workers [22] identified two related families of small elements by a bioinformatics analysis of the L. major genome sequence using as bait the "79-bp signature" common to trypanosomatid retroposons [21]. These families, named LmSIDER1 and LmSIDER2, contain 785 and 1073 copies per haploid genome, respectively. These authors raised a compelling hypothesis: these elements are extinct retroposons that have been recycled to accomplish regulatory functions for gene expression in Leishmania. Here, we describe the existence of this class of elements in the genome of $L$. infantum and other Leishmania species. The starting point of our work was the isolation from a macroarray of a clone showing strong hybridization signal when $L$. infantum total DNA was used as probe. Sequencing of this clone indicated that it contains a genomic fragment of chromosome 32 , but the bioinformatics analyses showed also that this clone would contain a repeated sequence because significant homology with different sequences located on the different $L$. infantum chromosomes was observed. After a thoughtful analysis, we identified a total of 54 elements in the L. infantum chromosome 32 and 27 elements in the chromosome 20. Sequence comparisons analysis between the repeated elements identified in this work with those described by Bringaud and co-workers in L. major, suggest that the elements described here belong to the SIDER2 family [22]. 
A

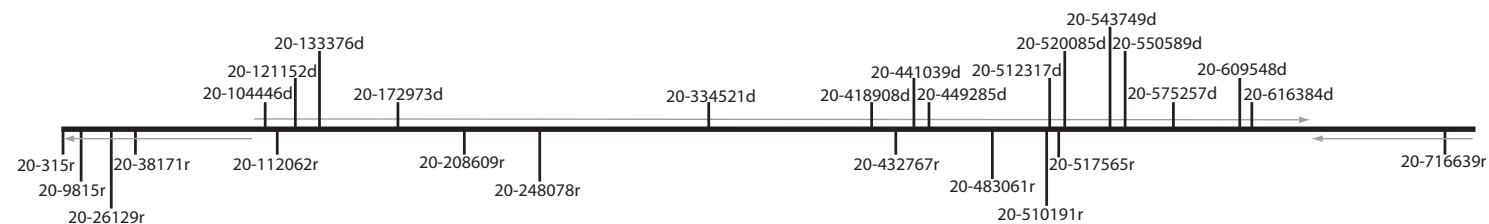

B

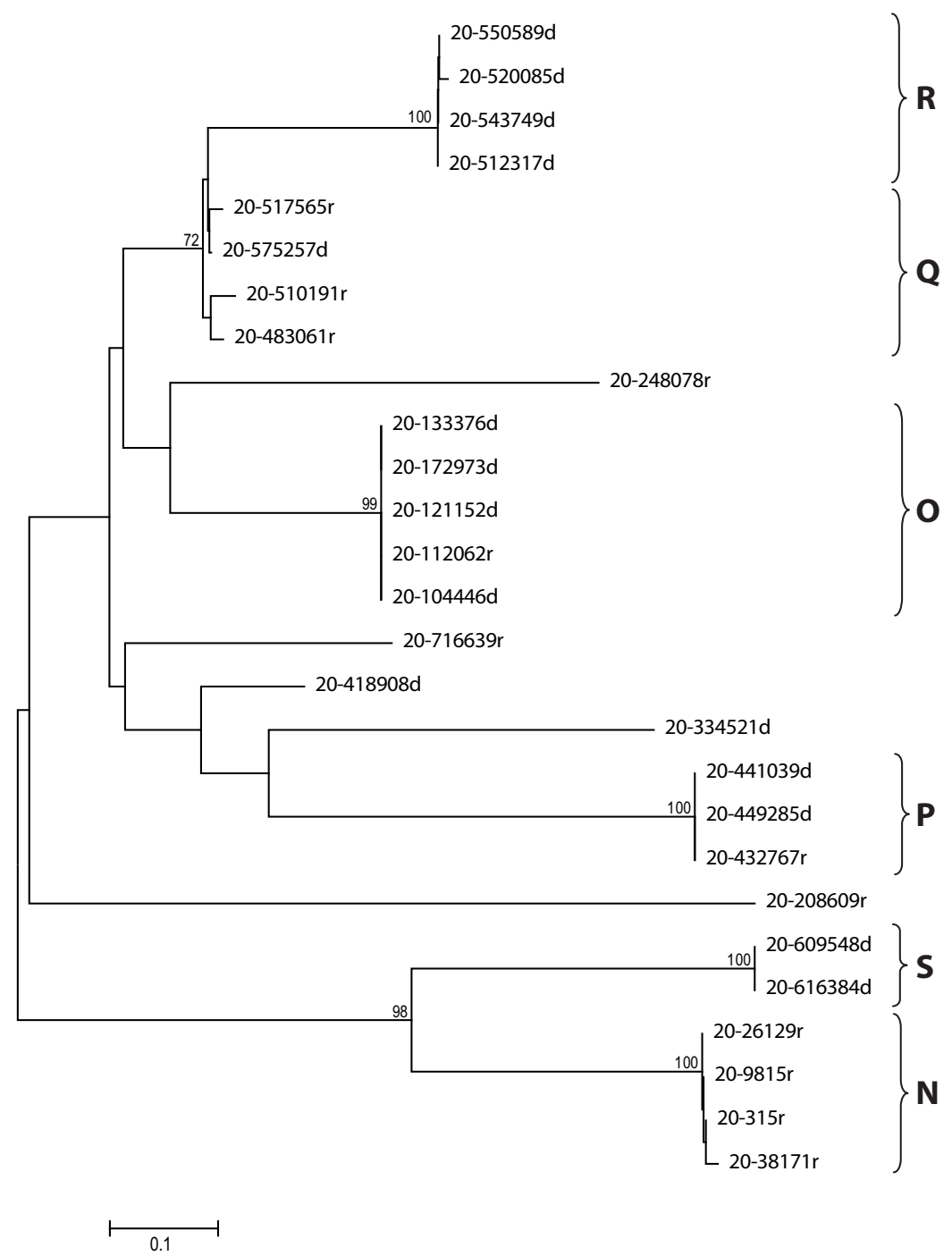

\section{Figure 4}

Analysis of SIDER2 elements of the $L$. infantum chromosome 20. (A) Genomic organization of LiSIDER2 elements, derived from the sequence of contig LinJ20_20070420_V3 [25]. (B) Phylogenetic tree of SIDER2 elements found in the L. infantum chromosome 20. Bootstrap values, expressed as percentages, were obtained from 1000 replicates (see legend to figure 2 for further details). The positions of the different subfamilies (N-S) are shown in the right margin. The scale represents number of changes per nucleotide. 
Table 3: SIDER elements present in the $L$. braziliensis chromosome 32

\begin{tabular}{|c|c|c|}
\hline Name $^{a}$ & Subfamily & Size (\%GC) \\
\hline $32-21338 r$ & $\mathrm{a}$ & $507(70.8)$ \\
\hline $32-33564 r$ & $\mathrm{a}$ & $429(70.2)$ \\
\hline $32-34297 r$ & $\mathrm{a}$ & $531(69.3)$ \\
\hline $32-46283 d$ & $\mathrm{a}$ & $503(69.8)$ \\
\hline $32-52976 r$ & $\mathrm{a}$ & $40 \mathrm{I}(70.3)$ \\
\hline $32-56914 r$ & $\mathrm{a}$ & $532(69.2)$ \\
\hline $32-8278 I d$ & $\mathrm{a}$ & $526(69.6)$ \\
\hline $32-190328 r$ & & $438(55.7)$ \\
\hline $32-218978 d$ & $\mathrm{a}$ & $465(72.5)$ \\
\hline $32-257472 d$ & & $565(66.4)$ \\
\hline $32-335085 r$ & $b$ & $422(66.4)$ \\
\hline $32-356555 d$ & $b$ & $388(67.3)$ \\
\hline $32-380245 d$ & $b$ & $422(67.5)$ \\
\hline $32-397315 d$ & $b$ & 340 (7I.2) \\
\hline $32-447556 \mathrm{~d}$ & & $499(57.7)$ \\
\hline $32-528 \mid 82 d$ & c & 459 (66.2) \\
\hline $32-558759 d$ & c & $629(63.3)$ \\
\hline $32-58 \mid I 22 r$ & c & $634(64.2)$ \\
\hline $32-632143 r$ & & $585(60.3)$ \\
\hline $32-640725 r$ & & $483(56.9)$ \\
\hline $32-693667 d$ & $d$ & $983(57.8)$ \\
\hline $32-695383 d^{*}$ & $d$ & $289(60.2)$ \\
\hline $32-716506 r$ & $d$ & 981 (58.0) \\
\hline $32-789725 r$ & e & $555(70.6)$ \\
\hline $32-799255 r$ & e & 550 (7I.I) \\
\hline $32-8165 \mid 2 r$ & $\mathrm{e}$ & 550 (7I.I) \\
\hline $32-819658 r$ & $\mathrm{e}$ & $550(70.7)$ \\
\hline $32-83606 \mathrm{Ir}$ & e & 569 (7I.9) \\
\hline $32-839556 r$ & e & 565 (7I..3) \\
\hline $32-842876 r$ & e & 565 (7I.7) \\
\hline $32-850687 r$ & e & 546 (7I.4) \\
\hline $32-867036 r$ & $f$ & $508(67.7)$ \\
\hline $32-875 \mid 42 r$ & $f$ & $486(67.7)$ \\
\hline $32-904 \mid 65 d$ & & $442(53.9)$ \\
\hline $32-965250 r$ & & $447(57.1)$ \\
\hline $32-97756 \mathrm{Ir}$ & & $540(60.2)$ \\
\hline $32-1033093 d$ & $g$ & $570(68.6)$ \\
\hline $32-1047782 r$ & $g$ & $571(67.6)$ \\
\hline $32-109140 \mathrm{Ir}$ & $\mathrm{h}$ & $604(67.1)$ \\
\hline $32-1121626 r$ & $\mathrm{~h}$ & $605(67.1)$ \\
\hline $32-13084 \mid 4 d$ & $\mathrm{i}$ & $523(64.4)$ \\
\hline $32-1332630 r$ & $\mathrm{i}$ & $528(64.2)$ \\
\hline $32-1396673 d$ & $\mathrm{i}$ & $496(64.5)$ \\
\hline $32-1539|4| d$ & j & $436(64.2)$ \\
\hline $32-1547986 d$ & $\mathrm{j}$ & $436(64.2)$ \\
\hline $32-1558134 d^{*}$ & $\mathrm{j}$ & $173(68.8)$ \\
\hline $32-1702406 d$ & $\mathrm{k}$ & 443 (63.9) \\
\hline $32-|7| 4067 d$ & $\mathrm{k}$ & $443(64.3)$ \\
\hline
\end{tabular}

aThe elements are named with the chromosome number (i.e., 32) and the position according the contig LbrM32 (version 2.0) sequence; $d$ and $r$ denote that the element is located on the plus or minus strand, respectively.

*Truncated element.

SIDER2 elements show outstanding features regarding genomic organization ([22]; this work): i) the elements are abundant and distributed along the different chromo- somes in all Leishmania species; ii) the elements constitutes subfamilies related in sequence and genomic vicinity; iii) the $L$. major and $L$. infantum SIDER2s are highly conserved both in sequence and chromosomal location. This degree of conservation in chromosomal location is not maintained between the L. major/L. infantum and L. braziliensis SIDER2s (at least for chromosome 32). It should be kept in mind that L. braziliensis is the most genetically and biologically divergent of the three species analyzed for this study [29]. A remarkable difference, which may be related with the variations in genomic distribution of SIDER2 elements among the Leishmania species, is that $L$. braziliensis possesses potentially active retrotransposons that are absent in the other two Leishmania species [6].

Accumulating data from different organisms do indicate that mobile elements and non-coding repetitive sequences are important elements in a genome and may be playing functional roles that vary from control of gene expression to chromosomal organization [1,3]. In this regard, the sequence features and genomic organization of SIDER2 elements are suggestive of relevant functional roles, but what kind of function can they be playing? The search for these elements within coding regions in $L$. infantum predicted genes indicates that no SIDER2 sequences are in coding region. The sole exception to this rule is the L. infantum database entry LinJ10_V3.1340, which contains sequence homology to SIDER2s. However, this entry is considered as pseudogene, since its sequence contains several in-frame stop codons. Remarkably, this putative pseudogene shows high sequence conservation with genes containing uninterrupted ORF in other kinetoplatids: LmjF10.1225 (L. major), LbrM10_V2.1350 (L. braziliensis), Tc00.1047053506153.6 (Trypanosoma cruzi) and Tb927.8.4690 (T. brucei). In spite of this particular finding, as overall conclusion, it must be stated that SIDER2 elements are rare in coding sequences.

On the other hand, several lines of evidence suggest that SIDER2 elements are frequently found in untranslated regions (UTRs) of genes, mainly 3'UTRs. Using both bioinformatics and experimental approaches, Bringaud et al. [22] demonstrated that SIDER2 elements are present in 3UTRs of many different genes. Furthermore, these authors showed experimental evidence that SIDER2 sequences are promoting downregulation of mRNA steady state levels. In addition, our database analyses showed that several $L$. chagasi cDNAs have SIDER2 sequences, reinforcing the idea that these elements are frequently found in UTRs of mRNAs, playing putative regulatory role in gene expression. 
A

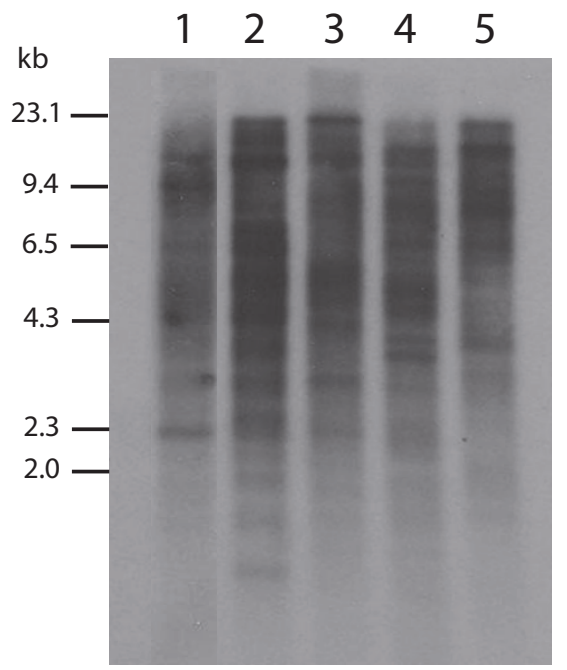

B

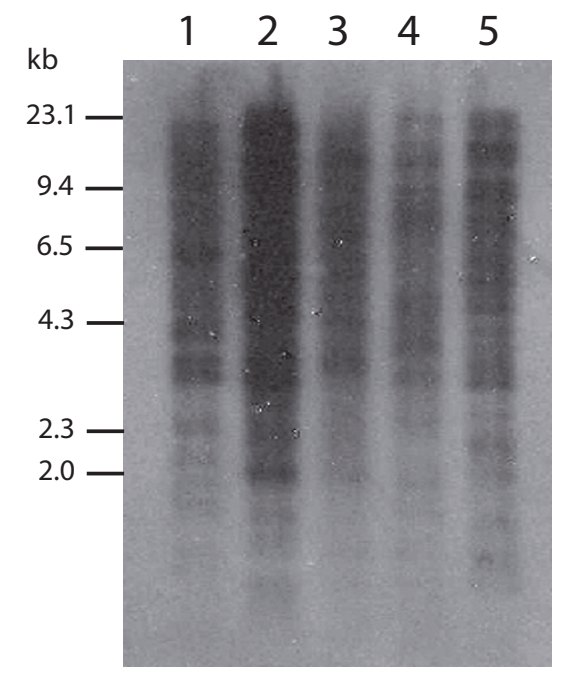

\section{Figure 5}

Southern blot analyses of the genomic distribution of SIDER2 sequences in different Leishmania species. Genomic DNA (Sall-digested) of L. infantum (lane I), L. major (lane 2), L. tropica (lane 3), L. mexicana (lane 4), and L. braziliensis (lane 5) was analyzed by Southern blotting using as probes either LiSIDER2-32-I2 I058r (panel A) or LiSIDER2-20-575257d (panel B).

Extrachromosomal DNA amplifications are commonly observed in different Leishmania species either after drug pressure or even in natural isolates $[30,31]$. When parasites are subjected to selective stresses, appropriate genomic DNA regions, containing flanking repeats, are amplified as extrachromosomal structures. According to the Beverley's model for explaining DNA amplification phenomena, the Leishmania genome should contain amplification-prone cassettes [30]. Thus, the genomic organization of SIDER2 elements in the Leishmania chromosomes (see Figs. 1 and 4) could be related with the amplification mechanism. Interestingly, another prediction of the model is the existence of two types of cassettes, those flanked by direct repeats and those flanked by inverted repeats. SIDER2 elements are found in both direct and inverted orientations, which further suggest their possible implication in Leishmania DNA amplification. In order to find additional cues supporting this idea, we looked for SIDER2 sequences in characterized DNA amplification structures of Leishmania. Remarkably, SIDER2 related sequences were found in several GenBank and EMBL entries corresponding to Leishmania DNA amplification structures. For example, three repeated sequences (RS1, RS2 and RS3) were identified in close proximity to the recombination points of extrachromosomal linear DNA amplicons M210 and M230 of L. major [32]. Schematic drawings for M210 and M230 amplicons, and for the genomic region of the source chromosome are depicted in figure 6A. Both amplicons have an inverted repeat structure, and the inversion occurred between repeats RS1 and RS2 for M210, and between RS2 and RS3 for M230. The three repeated sequences are 374-bp in size and show a high level of sequence identity (98\%) [32]. These repeated sequences have a remarkable homology with LiSIDER2 sequences (figure 6B), suggesting that they are members of an LmjSIDER2 subfamily. In other example, the repeated sequences, postulated to be involved in the formation of a linear amplicon in L. tarentolae [33], also share significant sequence homology with LiSIDER2 elements. These data suggest that indeed SIDER2 elements could be involved in the generation of some Leishmania extrachromosomal amplification.

Our search on GenBank and EMBL databases showed the existence of SIDER2 elements in other relevant Leishmania genomic regions. For example, homology to SIDER2 sequences is found in a $44-\mathrm{kb}$ genomic region, which was involved in mitotic stability of extrachromoses in L. donovani [34]. To date, the DNA elements participating in the chromosomal replication and segregation processes are largely unknown in Leishmania and other trypanosomatids. The difficulty to uncover the centromeres in trypanosomatids could be pointing to the existence of holocentric chromosomes that are characterized by the presence of a 
A

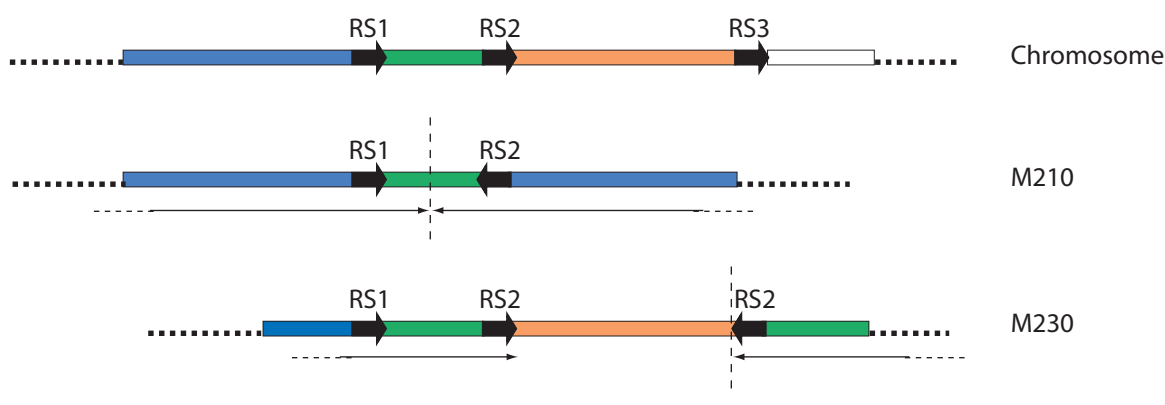

B

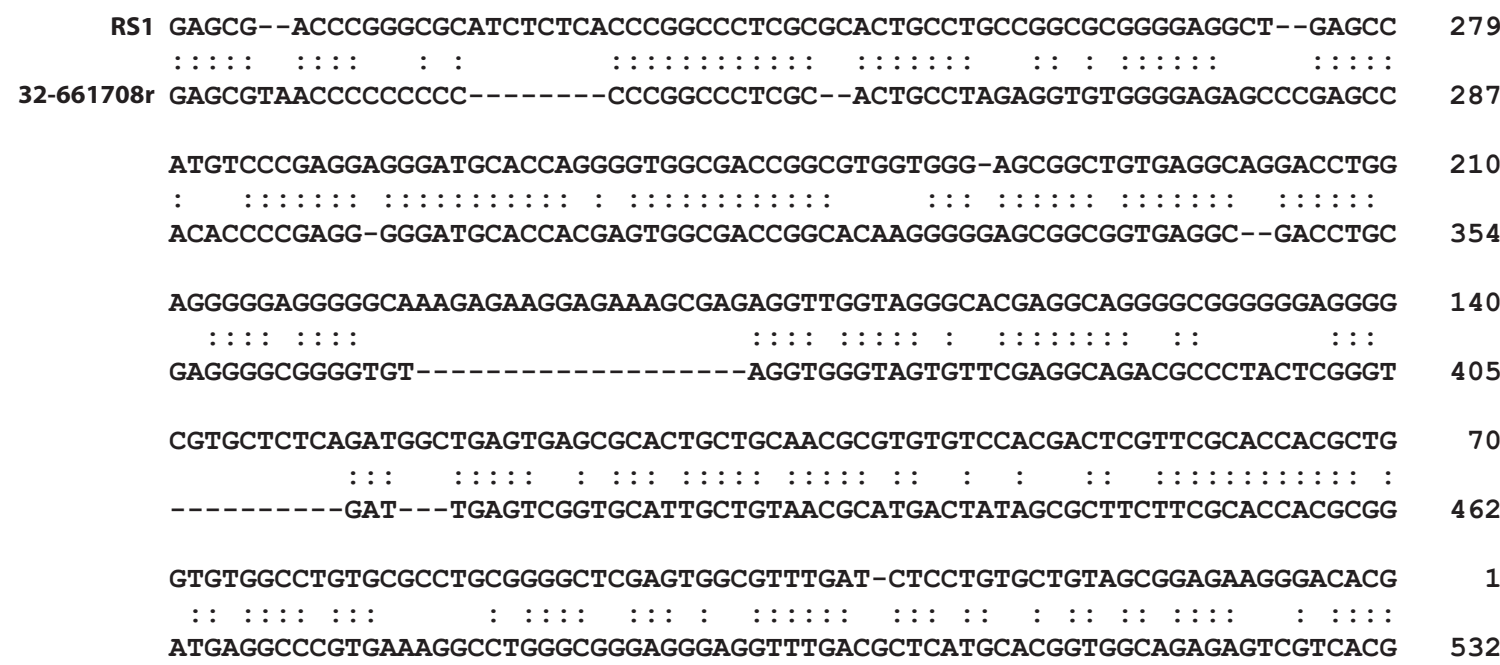

\section{Figure 6}

SIDER2 sequences are found in linear inverted DNA amplicons of Leishmania. (A) Schematic representation showing the location of the repeated sequences RSI, RS2 and RS3 in the M210 and M230 L. major amplicons and in the chromosome source (depicted according to data shown in reference [32]). The location and orientation of the RSI, RS2 and RS3 repeats are indicated by arrows. The rearrangement points are indicated by discontinuous, vertical lines. The extent and orientation of the inversions are indicated by horizontal arrows. The drawing is not to scale. (B) Alignment of RSI and LiSIDER2-32-66I708r sequences. Symbol (:) indicates identical bases in both sequences. Gaps (-) were introduced to maximize the alignment.

diffuse or nonlocalized centromere during mitosis [35]. In this scenario, SIDER2 elements should be considered as candidates for centromeric sequences. This hypothesis is based on two features of SIDER2 elements: they are distributed regularly along the chromosomes (Figs. 1 and 4 ) and they have $\mathrm{G}+\mathrm{C}$-rich sequences. Richness in $\mathrm{G}+\mathrm{C}$ sequences is observed in centromeres and pericentromic regions of many organisms [36]. Also, it is noticeable the existence, within the SIDER2 sequences, of G-rich tracts that are known for their propensity to form G-quadruplex DNA structures [37].

Finally, the presence of the "79-bp signature" in a large proportion of the SIDER2 elements may be suggestive of a transcriptional role for this class of repeats. In a previous report, we have demonstrated that the "79-bp signature" (also named Pr77), derived from T. cruzi L1Tc non-LTR retrotransposon has a RNA-pol II-dependent promoter that strongly activates gene transcription [38]. In this context, it may be postulated that SIDER2s bearing the "79-bp signature" could be acting as RNA-pol II recruiting points to enhance the transcriptional active at some chromosomal regions.

\section{Conclusion}

In this study, we describe several features of a family of novel repeated elements (named SIDER2) that are interspersed along the different chromosomes and present in 
all Leishmania species. We show an in-depth analysis of these elements in the $L$. infantum chromosomes 20 and 32 , and in the L. major and L. braziliensis chromosomes 32 . Apart from their proved role in post-transcriptional regulation of gene expression in Leishmania, our analyses suggest that SIDER2 elements could be involved in DNA amplification phenomena and, perhaps, they can represent centromeric sequences of holocentric chromosomes. In summary, SIDER2 elements constitute a relevant piece of the Leishmania genome organization, and this work provides a framework for investigating the functions of these sequences.

\section{Methods}

\section{Parasites and DNA isolation}

L. infantum JPC strain (MCAN/ES/98/LLM-724, clone M5) was used for arrays construction. For Southern blot analysis, the following Leishmania species were used: $L$. tropica (MHOM/SU/74/K-27), L. mexicana (MNYC/BZ/ 62/M-379), L. amazonensis (IFLA/BR/67/PH-8), L. braziliensis (MHOM/BR/75/M-2904) and L. major (MHOM/ $\mathrm{IL} / 80 /$ Friedlin). Promastigote forms were cultured in vitro at $26^{\circ} \mathrm{C}$ in RPMI 1640 medium (Sigma), supplemented with $10 \%$ heat-inactivated foetal calf serum (Sigma).

Genomic DNA was prepared from $2 \times 10^{8}$ promastigotes. After washing with phosphate-buffered saline (PBS), cells were suspended in $500 \mu \mathrm{l}$ of lysis buffer $(0.15 \mathrm{M} \mathrm{NaCl}, 0.1$ $M$ EDTA (pH 8.0) and 0.5\% SDS). Afterwards, proteinase $\mathrm{K}$ was added to a final concentration of $0.1 \mathrm{mg} / \mathrm{ml}$. After incubation for $30 \mathrm{~min}$ at $50^{\circ} \mathrm{C}$, samples were extracted sequentially with phenol, a phenol-chloroform-isoamyl alcohol (25:24:1) mixture and a chloroform-isoamyl alcohol (24:1) mixture. After adding 0.1 volumes of $3 \mathrm{M}$ sodium acetate and 2.5 volumes of cold ethanol, DNA was collected by centrifugation. The pellet was suspended in $200 \mu \mathrm{l}$ of Te buffer $(10 \mathrm{mM}$ Tris- $\mathrm{HCl}$ and $0.1 \mathrm{mM}$ EDTA, pH 8.0) and incubated with RNAse A $(20 \mu \mathrm{g} / \mathrm{ml}$ final concentration) for $30 \mathrm{~min}$ at $37^{\circ} \mathrm{C}$. Afterwards, DNA samples were extracted with a phenol-chloroform-isoamyl alcohol (25:24:1) mixture, and DNA precipitated by addition of 0.5 volumes of $7.5 \mathrm{M}$ ammonium acetate and 2 volumes of cold ethanol. Finally, DNA was suspended in $100 \mu \mathrm{l}$ of Te buffer.

\section{L. infantum genomic arrays}

Genomic DNA macroarrays were constructed as previously described [39]. Briefly, a genomic library of Sau3AI DNA fragments (4-kb average size) was constructed in pBluescript KS plasmid (Promega). DNA from individual colonies was prepared using the Perfectprep Plasmid 96 Vac kit (Eppendorf) and the BIOMEK 2000 robot (Beckam). DNA from 575 different clones was spotted in triplicate onto positively charged nylon membranes (Schleicher and Schuell) by NewBioTechnic (Sevilla, Spain).
Before hybridizations, macroarray membranes were washed with $0.5 \mathrm{M}$ phosphate buffer (pH 7.2) and incubated for $2 \mathrm{~h}$ at $65^{\circ} \mathrm{C}$ in $20 \mathrm{ml}$ of hybridization solution (0.5 M phosphate buffer ( $\mathrm{pH} 7.2$ ), 7\% SDS and $1 \mathrm{mM}$ EDTA). For hybridization, $350 \mathrm{ng}$ of $L$. infantum genomic DNA were labelled by nick-translation using $50 \mu \mathrm{Ci}$ of $[\alpha-$ $\left.{ }^{32} \mathrm{P}\right] \mathrm{dCTP}$ (3000 Ci/mmole; Amersham) and standard methods [40]. The labelled-DNA was added to the hybridization solution, and membranes were further incubated for $12 \mathrm{~h}$ at $65^{\circ} \mathrm{C}$. Afterwards, membranes were washed three times with washing solution $(40 \mathrm{mM}$ phosphate buffer (pH 7.2) and $0.1 \%$ SDS) for $20 \mathrm{~min}$ at $65^{\circ} \mathrm{C}$. Radioactive signals were analyzed by a Phosphorimager (Fuji BAS-1500).

\section{DNA sequencing of clone pGLi5-G8g}

Both strands of the insert of clone pGLi5-G8g were sequenced using an automated sequencer (ABI Prism 3730; Applied Biosystems) by the Genomics Unit of the Parque Científico de Madrid (SIDI-UAM). Nucleotide sequence of this clone has been deposited at European Molecular Biology Laboratory (EMBL/EBI) nucleotide sequence database under accession number AM937229.

\section{Identification of SIDER2 sequence elements in Leishmania databases}

An initial BLASTN search of the L. infantum database [25] using the sequence of clone pGLi5-G8g showed that this clone contains a genomic region from chromosome 32 . However, a subregion of approximately 550-bp was found to be widespread along the L. infantum genome. For the identification of these repeated sequences, now called LiSIDER2 elements, an iterative process was followed. For a given chromosome, sequence blocks showing sequence identity $\geq 60 \%$ and length $\geq 100$ nucleotides with pGLi5G8g sequence were considered for further analysis. Selected sequences (plus surrounding upstream and downstream sequences) were aligned using ClustalW. The clustering of sequences into subfamilies was carried out by phylogenetic analysis (see below). To determine the extent of the elements belonging to a given subfamily, the particular sequences were aligned with ClustalW and the extremities determined by visual inspection of the alignment. A subfamily was defined as a group of elements sharing sequence identity $\geq 85 \%$. When the size of an element was clearly different to the medium size for the elements of the subfamily, it was considered as truncated element. Each time a subfamily was identify, the sequence of the longest member of the subfamily was used to perform additional BLASTN searches in the Leishmania databases (contig sequences, [25]), the retrieved sequences (if new) were aligned as indicated above; the process was repeated until no new sequences were obtained. Finally, the remaining matches, non-assigned to any subfamily, were considered as SIDER2 "orphan" elements. Given the 
complexity of the identification process, we restricted the analyses to contigs for chromosomes 32 (LinJ32_20070420_V3) and 20 (LinJ20_20070420_V3).

Identification of SIDER2 elements in L. major and L. braziliensis databases [25] was performed by BLASTN searches using representative members for the LiSIDER2 subfamilies and the orphans LiSIDER2 elements of $L$. infantum chromosome 32. Each time a homologous sequence was retrieved, it was used to perform additional BLASTN searches in the database. The size and genomic positions for the different LmjSIDER2 or LbSIDER2 elements were determined by sequence alignments using ClustalW and manual corrections. Again, we restricted our analysis to chromosomes 32 of $L$. major (LmjF32_01_20050601_V5.2) and L. braziliensis (LbrM32, version 2.0).

\section{Multiple alignments and phylogenetic trees}

The complete LiSIDER2 sequences were aligned using the default options of ClustalW2 [41]. The resulting alignments were used to perform phylogenetic analysis conducted with the program MEGA version 3.1 [42] using the Neighbour-Joining method and default parameters.

\section{Other databases mining}

The different LiSIDER2 elements found in L. infantum chromosome 32 were used for BLASTN searches in T. brucei and T. cruzi databases [25]. Also, BLAST searches were performed in GenBank and EMBL databases.

\section{DNA probes and Southern blot analysis}

LiSIDER2-32-121058r and LiSIDER2-20-575257d elements were PCR amplified using as template genomic DNA from $L$. infantum JPC strain. As primers, the following oligonucleotides were used: LiRS-32-Ad (5'CCGCCCCGAAATATAAGT-3') and LiRS-32-Ar (5'GCCTCCATGCGCGGTGTC-3') for LiSIDER2-32121058r; 20R-d (5'-CCACATCGCGCGTGGCGC-3') and 20R-r (5'-TGACGTGTGGACCCCGCT-3') for LiSIDER2$20-575257 \mathrm{~d}$. The amplification products were cloned into the pCR2. 1 vector (Invitrogen), yielding clones pLiRS-32A (LiSIDER2-32-121058r) and pLiRS-20Q (LiSIDER2-20$575257 \mathrm{~d})$. The authenticity of clones and the fidelity of the PCR-amplification were verified by nucleotide sequencing.

For Southern blot analysis, $1 \mu \mathrm{g}$ of total DNA from the different Leishmania species was digested with the SalI restriction enzyme and electrophoresed on $0.8 \%$ agarose cells. After ethidium bromide visualization, DNA was transferred to nylon membranes (Hybond-N, Amersham) by standard methods [43]. For probe preparations, EcoRIinserts of clones pLiRS-32A and pLiRS-20Q were labelled with [ $\alpha$-32P]dCTP by nick-translation [43]. Hybridizations were performed as reported earlier [44].

\section{Authors' contributions}

CF, MCL and MCT carried out the different steps of macroarrays construction. CF performed hybridization of macroarrays, PCR amplification and Southern blotting. JMR conceived the project, supervised the experiments and performed the bioinformatics analyses. MCL and MCT helped to draft the manuscript. CF prepared the final version of figures. JMR wrote the final version of the manuscript. All authors have read and approved the final manuscript.

\section{Additional material}

\section{Additional file 1}

ClustalW2 alignment of the 54 LiSIDER2 sequences present in the $\mathrm{L}$. infantum chromosome 32.

Click here for file

[http://www.biomedcentral.com/content/supplementary/1471-

2164-9-263-S1.doc]

\section{Additional file 2}

ClustalW2 alignment of the 27 LiSIDER2 sequences present in the $\mathrm{L}$. infantum chromosome 20.

Click here for file

[http://www.biomedcentral.com/content/supplementary/14712164-9-263-S2.doc]

\section{Acknowledgements}

Genome sequence data from the Sanger Institute sequencing projects (GeneDB) were invaluable for this work and their provision in the public domain is gratefully acknowledged. Thanks are also given to three anonymous reviewers for their valuable comments. Researchers interested in the sequence datasets described here should contact the authors. This work was funded by grants from the Ministerio de Ciencia y Tecnología (BFU2006-08346), the Instituto de Salud Carlos III (ISCIII-RETIC RD06/ 002 I/0008-FEDER and ISCIII-RETIC RD06/002I/00 I4-FEDER), and Plan Nacional de I+D+I (BFU2007-65095). Also, an institutional grant from Fundación Ramón Areces is acknowledged.

\section{References}

I. Kazazian $\mathrm{HH}$ Jr.: Mobile elements: drivers of genome evolution. Science 2004, 303(5664): I626-1632.

2. Kapitonov VV, Jurka J: Molecular paleontology of transposable elements in the Drosophila melanogaster genome. Proc Natl Acad Sci U S A 2003, 100 (I I):6569-6574.

3. Slotkin RK, Martienssen R: Transposable elements and the epigenetic regulation of the genome. Nat Rev Genet 2007, 8(4):272-285.

4. Desjeux P: Leishmaniasis: current situation and new perspectives. Comp Immunol Microbiol Infect Dis 2004, 27(5):305-3 I8.

5. Ivens AC, Peacock CS, Worthey EA, Murphy L, Aggarwal G, Berriman M, Sisk E, Rajandream MA, Adlem E, Aert R, Anupama A, Apostolou Z, Attipoe P, Bason N, Bauser C, Beck A, Beverley SM, Bianchettin G, Borzym K, Bothe G, Bruschi CV, Collins M, Cadag E, Ciarloni L, Clayton C, Coulson RM, Cronin A, Cruz AK, Davies RM, De Gaudenzi J, Dobson DE, Duesterhoeft A, Fazelina G, Fosker N, Frasch AC, Fraser A, Fuchs M, Gabel C, Goble A, Goffeau A, Harris D, Hertz-Fowler C, 
Hilbert H, Horn D, Huang Y, Klages S, Knights A, Kube M, Larke N, Litvin L, Lord A, Louie T, Marra M, Masuy D, Matthews K, Michaeli S, Mottram JC, Muller-Auer S, Munden H, Nelson S, Norbertczak H, Oliver K, O'Neil S, Pentony M, Pohl TM, Price C, Purnelle B, Quail MA, Rabbinowitsch E, Reinhardt R, Rieger M, Rinta J, Robben J, Robertson L, Ruiz JC, Rutter S, Saunders D, Schafer M, Schein J, Schwartz DC, Seeger K, Seyler A, Sharp S, Shin H, Sivam D, Squares R, Squares S, Tosato V, Vogt C, Volckaert G, Wambutt R, Warren T, Wedler H, Woodward J, Zhou S, Zimmermann W, Smith DF, Blackwell JM, Stuart KD, Barrell B, Myler PJ: The Genome of the Kinetoplastid Parasite, Leishmania major. Science 2005, 309(5733):436-442.

6. Peacock CS, Seeger K, Harris D, Murphy L, Ruiz JC, Quail MA, Peters N, Adlem E, Tivey A, Aslett M, Kerhornou A, Ivens A, Fraser A, Rajandream MA, Carver T, Norbertczak H, Chillingworth T, Hance Z, Jagels K, Moule S, Ormond D, Rutter S, Squares R, Whitehead S, Rabbinowitsch E, Arrowsmith C, White B, Thurston S, Bringaud F, Baldauf SL, Faulconbridge A, Jeffares D, Depledge DP, Oyola SO, Hilley JD, Brito LO, Tosi LRO, Barrell B, Cruz AK, Mottram JC, Smith DF, Berriman M: Comparative genomic analysis of three Leishmania species that cause diverse human disease. Nat Genet 2007, 39(7):839-847.

7. Smith DF, Peacock CS, Cruz AK: Comparative genomics: From genotype to disease phenotype in the leishmaniases. Int J Parasitol 2007, 37(I I): I I73-I I86.

8. El-Sayed NM, Myler PJ, Blandin G, Berriman M, Crabtree J, Aggarwal G, Caler E, Renauld H, Worthey EA, Hertz-Fowler C, Ghedin E, Peacock C, Bartholomeu DC, Haas BJ, Tran AN, Wortman JR, Alsmark UC, Angiuoli S, Anupama A, Badger J, Bringaud F, Cadag E, Carlton JM, Cerqueira GC, Creasy T, Delcher AL, Djikeng A, Embley TM, Hauser C, Ivens AC, Kummerfeld SK, Pereira-Leal JB, Nilsson D, Peterson J, Salzberg SL, Shallom J, Silva JC, Sundaram J, Westenberger S, White O, Melville SE, Donelson JE, Andersson B, Stuart KD, Hall $\mathrm{N}$ : Comparative genomics of trypanosomatid parasitic protozoa. Science 2005, 309(5733):404-409.

9. Martinez-Calvillo S, Yan S, Nguyen D, Fox M, Stuart K, Myler PJ: Transcription of Leishmania major Friedlin chromosome I initiates in both directions within a single region. Mol Cell 2003, I I(5): I29|-1299.

10. Requena JM, Lopez MC, Alonso C: Genomic repetitive DNA elements of Trypanosoma cruzi. Parasitol Today 1996, 12:279-283.

II. Wickstead B, Ersfeld K, Gull K: Repetitive elements in genomes of parasitic protozoa. Microbiol Mol Biol Rev 2003, 67(3):360-75, table of contents.

12. El-Sayed NM, Myler PJ, Bartholomeu DC, Nilsson D, Aggarwal G, Tran AN, Ghedin E, Worthey EA, Delcher AL, Blandin G, Westenberger SJ, Caler E, Cerqueira GC, Branche C, Haas B, Anupama A, Arner E, Aslund L, Attipoe $\mathrm{P}$, Bontempi E, Bringaud F, Burton $\mathrm{P}$ Cadag E, Campbell DA, Carrington M, Crabtree J, Darban H, da Silveira JF, de Jong P, Edwards K, Englund PT, Fazelina G, Feldblyum T, Ferella M, Frasch AC, Gull K, Horn D, Hou L, Huang Y, Kindlund E, Klingbeil M, Kluge S, Koo H, Lacerda D, Levin MJ, Lorenzi H, Louie T, Machado CR, McCulloch R, McKenna A, Mizuno Y, Mottram JC, Nelson S, Ochaya S, Osoegawa K, Pai G, Parsons M, Pentony M, Pettersson U, Pop M, Ramirez JL, Rinta J, Robertson L, Salzberg SL, Sanchez DO, Seyler A, Sharma R, Shetty J, Simpson AJ, Sisk E, Tammi MT, Tarleton R, Teixeira S, Van Aken S, Vogt C, Ward PN, Wickstead B, Wortman J, White O, Fraser CM, Stuart KD, Andersson B: The genome sequence of Trypanosoma cruzi, etiologic agent of Chagas disease. Science 2005, 309(5733):409-4I5.

13. Ellis J, Crampton J: Characterisation of a simple, highly repetitive DNA sequence from the parasite Leishmania donovani. Mol Biochem Parasitol 1988, 29:9-18.

14. Howard MK, Kelly JM, Lane RP, Miles MA: A sensitive repetitive DNA probe that is specific to the Leishmania donovani complex and its use as an epidemiological and diagnostic reagent. Mol Biochem Parasitol 1991, 44:63-72.

15. Piarroux R, Azaiez R, Lossi AM, Reynier P, Muscatelli F, Gambarelli F, Fontes $M$, Dumon $H$, Quilici M: Isolation and characterization of a repetitive DNA sequence from Leishmania infantum: development of a visceral leishmaniasis polymerase chain reaction. Am J Trop Med Hyg 1993, 49(3):364-369.

16. Ravel C, Wincker P, Bastien P, Blaineau C, Pages M: A polymorphic minisatellite sequence in the subtelomeric regions of chromosomes I and $\mathbf{V}$ in Leishmania infantum. Mol Biochem Parasitol 1995, 74(I):3|-4I.
17. Requena JM, Soto M, Quijada L, Carrillo G, Alonso C: A region containing repeated elements is associated with transcriptional termination of Leishmania infantum ribosomal RNA genes. Mol Biochem Parasitol 1997, 84(I): I0I-IIO.

18. Fu G, Barker DC: Characterisation of Leishmania telomeres reveals unusual telomeric repeats and conserved telomereassociated sequence. Nucleic Acids Res 1998, 26(9):2161-2I67.

19. Sunkin SM, Kiser P, Myler PJ, Stuart K: The size difference between leishmania major friedlin chromosome one homologues is localized to sub-telomeric repeats at one chromosomal end. Mol Biochem Parasitol 2000, 109(I): I-I5

20. Dumas C, Chow C, Muller M, Papadopoulou B: A novel class of developmentally regulated noncoding RNAs in Leishmania. Eukaryot Cell 2006, 5(I 2):2033-2046.

21. Bringaud F, Ghedin E, Blandin G, Bartholomeu DC, Caler E, Levin MJ, Baltz T, El-Sayed NM: Evolution of non-LTR retrotransposons in the trypanosomatid genomes: Leishmania major has lost the active elements. Mol Biochem Parasitol 2006, I 45(2): I 58-I70.

22. Bringaud F, Muller M, Cerqueira GC, Smith M, Rochette A, El-Sayed NMA, Papadopoulou B, Ghedin E: Members of a large retroposon family are determinants of post-transcriptional gene expression in Leishmania. PLoS Pathog 2007, 3(9): |29|-| 307.

23. Boucher N, Wu Y, Dumas C, Dube M, Sereno D, Breton M, Papadopoulou $B$ : A common mechanism of stage-regulated gene expression in Leishmania mediated by a conserved 3'. untranslated region element. I Biol Chem 2002, 277(22):195II-19520.

24. McNicoll F, Muller M, Cloutier S, Boilard N, Rochette A, Dube M, Papadopoulou B: Distinct 3'-untranslated region elements regulate stage-specific mRNA accumulation and translation in Leishmania. J Biol Chem 2005, 280(42):35238-35246.

25. GeneDB [http://www.genedb.org/]

26. Bringaud F, Garcia-Perez JL, Heras SR, Ghedin E, El-Sayed NM, Andersson B, Baltz T, Lopez MC: Identification of non-autonomous non-LTR retrotransposons in the genome of Trypanosoma cruzi. Mol Biochem Parasitol 2002, I 24(I-2):73-78.

27. Pedrosa AL, Silva AM, Ruiz JC, Cruz AK: Characterization of LSTR533: uncovering a novel repetitive element in Leishmania. Int J Parasitol 2006, 36(2):21I-2I7.

28. Clayton C, Adams M, Almeida R, Baltz T, Barrett M, Bastien P, Belli S, Beverley S, Biteau N, Blackwell J, Blaineau C, Boshart M, Bringaud F, Cross G, Cruz A, Degrave W, Donelson J, El-Sayed N, Fu G, Ersfeld K, Gibson W, Gull K, Ivens A, Kelly J, Lawson D, Lebowitz J, Majiwa P, Matthews K, Melville S, Merlin G, Michels P, Myler P, Norrish A, Opperdoes F, Papadopoulou B, Parsons M, Seebeck T, Smith D, Stuart K, Turner M, Ullu E, Vanhamme L: Genetic nomenclature for Trypanosoma and Leishmania. Mol Biochem Parasitol 1998, 97(I2):221-224.

29. Bañuls AL, Hide M, Prugnolle F: Leishmania and the leishmaniases: a parasite genetic update and advances in taxonomy, epidemiology and pathogenicity in humans. Adv Parasitol 2007, 64:1-109.

30. Beverley SM: Gene amplification in Leishmania. Annu Rev Microbiol I99|, 45:4I7-444.

31. Segovia M, Ortiz G: LDI amplifications in Leishmania. Parasitol Today 1997, 13:342-348.

32. Ortiz G, Segovia M: Characterisation of the novel junctions of two minichromosomes of Leishmania major. Mol Biochem Parasitol |996, 82(2): |37-| 44.

33. Genest PA, ter Riet B, Dumas C, Papadopoulou B, van Luenen HGAM, Borst P: Formation of linear inverted repeat amplicons following targeting of an essential gene in Leishmania. Nucleic Acids Res 2005, 33(5): 1699- 1709.

34. Dubessay $P$, Ravel $C$, Bastien $P$, Lignon MF, Ullman B, Pages M, Blaineau C: Effect of large targeted deletions on the mitotic stability of an extra chromosome mediating drug resistance in Leishmania. Nucleic Acids Res 200I, 29(15):323I-3240.

35. Villasante A, Abad JP, Mendez-Lago M: Centromeres were derived from telomeres during the evolution of the eukaryotic chromosome. Proc Natl Acad Sci U S A 2007, 104(25): 10542-10547.

36. Abad JP, Carmena M, Baars S, Saunders RD, Glover DM, Ludena P, Sentis C, Tyler-Smith C, Villasante A: Dodeca satellite: a conserved G+C-rich satellite from the centromeric heterochromatin of Drosophila melanogaster. Proc Natl Acad Sci U S A 1992 89(10):4663-4667. 
37. Burge S, Parkinson GN, Hazel P, Todd AK, Neidle S: Quadruplex DNA: sequence, topology and structure. Nucleic Acids Res 2006, 34(19):5402-54I5.

38. Heras SR, Lopez MC, Olivares M, Thomas MC: The LITc non-LTR retrotransposon of Trypanosoma cruzi contains an internal RNA-pol II-dependent promoter that strongly activates gene transcription and generates unspliced transcripts. Nucleic Acids Res 2007, 35(7):2199-2214.

39. Quijada L, Soto M, Requena JM: Genomic DNA macroarrays as a tool for analysis of gene expression in Leishmania. Exp Parasitol 2005, I I I (I):64-70.

40. Sambrook J, Russel DW: The condensed protocols from molecular cloning: a laboratory manual. Cold Spring Harbor Laboratory Press 2006, Cold Spring Harbor:New York.

41. European Bioinformatics Institute Site Index [http:// www.ebi.ac.uk/services/]

42. Kumar S, Tamura K, Nei M: MEGA3: Integrated software for Molecular Evolutionary Genetics Analysis and sequence alignment. Brief Bioinform 2004, 5(2): 150-163.

43. Sambrook J, Fritsch EF, Maniatis T: Molecular Cloning: A Laboratory Manual. 2nd Edn Cold Spring Harbor Laboratory Press, Cold Spring Harbor, NY 1989.

44. Quijada L, Soto M, Alonso C, Requena JM: Analysis of post-transcriptional regulation operating on transcription products of the tandemly linked Leishmania infantum hsp70 genes. J Biol Chem 1997, 272(7):4493-4499.

45. National Center for Biotechnology Information BLAST tool [http://www.ncbi.nlm.nih.gov/blast/Blast.cgi]

Publish with Bio Med Central and every scientist can read your work free of charge

"BioMed Central will be the most significant development for disseminating the results of biomedical research in our lifetime. "

Sir Paul Nurse, Cancer Research UK

Your research papers will be:

- available free of charge to the entire biomedical community

- peer reviewed and published immediately upon acceptance

- cited in PubMed and archived on PubMed Central

- yours - you keep the copyright

Submit your manuscript here:

http://www.biomedcentral.com/info/publishing_adv.asp
BiolMedcentral 